\title{
Influence of interface inhomogeneities in thin-film Schottky diodes
}

DOI:

$10.1063 / 1.5004247$

Document Version

Accepted author manuscript

Link to publication record in Manchester Research Explorer

\section{Citation for published version (APA):}

Wilson, J., Zhang, J., Li, Y., Wang, Y., Xin, Q., \& Song, A. (2017). Influence of interface inhomogeneities in thinfilm Schottky diodes. Applied Physics Letters, 111(21), [213503]. https://doi.org/10.1063/1.5004247

\section{Published in:}

Applied Physics Letters

\section{Citing this paper}

Please note that where the full-text provided on Manchester Research Explorer is the Author Accepted Manuscript or Proof version this may differ from the final Published version. If citing, it is advised that you check and use the publisher's definitive version.

\section{General rights}

Copyright and moral rights for the publications made accessible in the Research Explorer are retained by the authors and/or other copyright owners and it is a condition of accessing publications that users recognise and abide by the legal requirements associated with these rights.

\section{Takedown policy}

If you believe that this document breaches copyright please refer to the University of Manchester's Takedown Procedures [http://man.ac.uk/04Y6Bo] or contact uml.scholarlycommunications@manchester.ac.uk providing relevant details, so we can investigate your claim.

\section{OPEN ACCESS}




\title{
Influence of interface inhomogeneities in thin-film Schottky diodes
}

\author{
Joshua Wilson, ${ }^{1}$ Jiawei Zhang, ${ }^{1}$ Yunpeng $\mathrm{Li},{ }^{2}$ Yiming Wang, ${ }^{2}$ Qian Xin, ${ }^{2}$ and Aimin Song ${ }^{1,2, *}$ \\ ${ }^{1}$ School of Electrical and Electronic Engineering, \\ University of Manchester, Manchester, M13 9PL, United Kingdom \\ ${ }^{2}$ School of Physics, Shandong University, Jinan 250100, People's Republic of China
}

(Dated: October 28, 2017)

\begin{abstract}
The scalability of thin-film transistors has been well documented, but there have been very few investigations into of the effects of device scalability in Schottky diodes. Indium-gallium-zinc-oxide (IGZO) Schottky diodes were fabricated with IGZO thicknesses of 50, 150 and $250 \mathrm{~nm}$. Despite the same IGZO-Pt interface and Schottky barrier being formed in all devices, reducing the IGZO thickness caused a dramatic deterioration of the current-voltage characteristics, most notably increasing the reverse current by nearly five orders of magnitude. Furthermore, the forward characteristics display an increase in ideality factor and a reduction in barrier height. The origins of this phenomenon have been elucidated using device simulations. Firstly, when the semiconductor layer is fully depleted, the electric field increases with reducing thickness, leading to an increased diffusion current. However, the effects of diffusion only offer a small contribution to the huge variations in reverse current seen in the experiments. To fully explain this effect the role of inhomogeneities in the Schottky barrier height has been considered. Contributions from lower barrier regions (LBRs) are found to dominate the reverse current. The conduction band minimum below these LBRs is strongly dependent upon thickness and bias, leading to reverse current variations as large as several orders of magnitude. Finally, it is demonstrated that the thickness dependence of the reverse current is exacerbated as the magnitude of the inhomogeneities is increased, and alleviated in the limit where the LBRs are large enough not to be influenced by the adjacent higher barrier regions.
\end{abstract}

In recent years, efforts to realise large-area flexible thinfilm electronics have branched out to incorporate the use of a large variety of materials [1-3]. Oxide semiconductors, particularly indium-gallium-zinc-oxide (IGZO), have emerged as leading candidates for application in future display technology [4]. IGZO has demonstrated excellent mobility and transparency [5], and can also be deposited at room temperature, making it compatible with flexible substrates [6]. Initially, most studies were focussed on thin-film transistors (TFTs) [7, 8]. However, there is now a growing body of literature on IGZO Schottky diodes [9-12]. These efforts have led to the realisation of excellent electronic properties such as rectification ratios $>10^{7}$, barrier heights $>0.9 \mathrm{eV}$ and ideality factors close to unity. Notable achievements include fabricating devices on flexible substrates [9], exhibiting gigahertz operating frequencies $[12,13]$ and the combination of these two [14], thus demonstrating the potential of IGZO Schottky diodes for applications in future mobile technology. IGZO Schottky junctions have also found use in other thin-film device architectures and applications including metal-semiconductor field-effect transistors (MESFETs) [15], memory storage $[16,17]$ and energy harvesting [18].

Unlike TFTs, where scaling phenomena such as the short-channel effect are well documented [19, 20], the effects of device scalability on IGZO Schottky diodes has received limited attention. So far, studies of IGZO Schottky diodes have been focused on the forward current, except work regarding the reverse breakdown voltage [21].

* Please address correspondence to A.Song@manchester.ac.uk
Reducing the thickness of the IGZO should be beneficial for the rectification ratio - because of an increase in the forward current - as well as for potential device flexibility due to a reduction in tensile stress for a fixed radius of curvature. However, some studies show unexplained evidence of a deterioration of the reverse current with reducing thickness [14, 21].

In this work, the significant effects of scaling IGZO thickness upon the current-voltage $(J-V)$ characteristics of thin-film Schottky diodes are presented. It was found that the reverse current was drastically dependent on the IGZO thickness. With the help of device simulations, the cause of this dependence is elucidated and attributed to the inhomogeneous nature of the Schottky barrier height $\left(\Phi_{B}\right)$. Finally, the effects of variations in the magnitude and area of the barrier inhomogeneities on the reverse current are discussed.

The inset of Fig. 1b shows the structure of the Pt-IGZO Schottky diodes fabricated in this work. $\mathrm{SiO}_{2}-\mathrm{Si}$ wafers were cleaned by sonic agitation in an ultrasonic bath using DECON 90, de-ionized water, acetone and isopropyl alcohol, respectively. Using radio-frequency sputtering with $\mathrm{Ar}$ as the working gas, a $100 \mathrm{~nm}$ thick Pt layer was deposited as the Schottky contact. In order to more easily and clearly replicate the thickness dependence, the decision was made not to carry out oxygen treatment of the Schottky contact, a well-documented requirement for optimising the barrier height of oxide semiconductor Schottky diodes $[22,23]$. This is because the in optimised IGZO-Pt Schottky diodes the reverse current is often near the resolution limit of the measurement equipment. Following the $\mathrm{Pt}$ deposition, an IGZO layer was sputtered using an IGZO target with a molar ratio of 

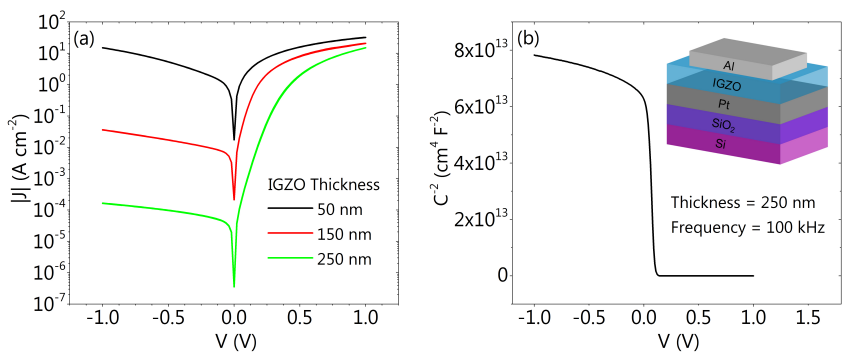

FIG. 1. (a) $|J|-V$ characteristics of devices with IGZO thicknesses of 50, 150 and $250 \mathrm{~nm}$. (b) $C^{-2}-V$ curve for the $250 \mathrm{~nm}$ thick IGZO diode from which the carrier density $N_{D}$ was extracted. The inset is the structure of the fabricated IGZO-Pt Schottky diodes.

1:1:2 $\left(\mathrm{In}_{2} \mathrm{O}_{3}: \mathrm{Ga}_{2} \mathrm{O}_{3}: \mathrm{ZnO}\right)$ under the same conditions as used for Pt. Three different thicknesses of IGZO were chosen: 50, 150 and $250 \mathrm{~nm}$. Al was chosen as the ohmic contact and was deposited via thermal evaporation and patterned using shadow masks. The $J-V$ properties were measured using an Agilent E5260B semiconductor analyzer. Capacitance-voltage $(C-V)$ measurements were carried out at $100 \mathrm{kHz}$ using Agilent E4980A LCR meter, this high frequency was used as a precaution against the influence of electron traps.

The resulting $|J|-V$ characteristics, shown in Fig. 1a, display a drastic thickness dependence despite the same IGZO-Pt Schottky barrier being present in all devices. The most notable change is the huge increase in reverse current; nearly five orders of magnitude for a $200 \mathrm{~nm}$ reduction in IGZO thickness, amounting to a sharp degradation in the quality of the diodes. Hence, the rectification ratio fell from $9.0 \times 10^{4}$ in the $250 \mathrm{~nm}$ diodes to only 2.2 in $50 \mathrm{~nm}$ diodes. Similar dependencies on reverse current can be seen (without explanation) in Fig. If of [14] and Fig. 2a of [21], where the authors focused on frequency response and reverse breakdown voltage, respectively. Furthermore, there is a significant increase in ideality factor, $n$, and a major reduction in $\Phi_{B}$ as thickness is reduced. For example, in the diodes with $250 \mathrm{~nm}$ of IGZO, $\Phi_{B}=0.64 \mathrm{eV}$ and $n=1.40$, but for diodes with $150 \mathrm{~nm}$ of IGZO, $\Phi_{B}=0.51 \mathrm{eV}$ and $n=1.48$. The sharp deterioration of the $|J|-V$ characteristics meant that no fitting was possible for the $50 \mathrm{~nm}$ devices. An explanation for this thickness dependence is not possible using thermionic emission theory:

$$
J=A^{*} T^{2} \exp \left(-\frac{\Phi_{B}}{k T}\right)\left[\exp \left(\frac{q V}{n k T}\right)-1\right]
$$

where $A^{*}=\frac{4 \pi q m^{*} k^{2}}{h^{3}}$ is the Richardson constant $\left(\approx 41 \mathrm{~A} \mathrm{~cm}^{-2} \mathrm{~K}^{-2}\right.$ in IGZO), $m^{*}$ is the effective mass of an electron, $k$ is the Boltzmann constant, $q$ is the fundamental charge, $h$ is the Planck constant, $V$ is the applied bias and $T$ is the temperature. According to this equation, the current should only be dependent upon $\Phi_{B}$ and $V$ (for a set material at room temperature). In addi- tion, thermionic emission theory presupposes a flat reverse current, but between $-0.2 \mathrm{~V}$ and $-1 \mathrm{~V}$ the reverse current increases by $636 \%, 265 \%$ and $169 \%$ for the 50 , 150 and $250 \mathrm{~nm}$ thick cases, respectively. Even when considering image force effects, such a thickness dependence would not be expected [24]. The only anticipated thickness dependence in the experimental data is that the current in the series resistance limited regime (at higher forward bias) increased with reducing thickness, explainable by the fact that thinner films offer less resistance.

In the interest of clarifying the possible origins of this behaviour, two-dimensional device simulations were carried out using Silvaco Atlas. The modelled diodes have a width of $100 \mathrm{~nm}$ and semiconductor thicknesses of 50, 150 and $250 \mathrm{~nm}$. The Schottky barrier height was set in accordance with the results extracted from the $|J|$ $V$ curve of the $250 \mathrm{~nm}$ thick diode $(0.64 \mathrm{eV})$ and the doping was set in accordance with the experimental results extracted from the $C^{-2}-V$ curve in Fig. 1b, i.e., $N_{D}=6 \times 10^{13} \mathrm{~cm}^{-3}$. The default IGZO parameters were used as outlined in [25]. The mesh density within the IGZO layer was finer nearer the Schottky interface and was fixed for all thicknesses.

Figure 2a shows the simulated $|J|-V$ characteristics of diodes with different thicknesses of IGZO. In this case, there is a clear trend showing that for reducing thickness there is an increase in reverse current, but only by less than one order of magnitude, which is inconsistent with the experimental results. The comparatively small variation in reverse current can be accounted for by considering the diffusion equation [24]:

$$
J \approx q \mu N_{C} \mathcal{E}_{\text {max }} \exp \left(-\frac{\Phi_{B}}{k T}\right)\left[\exp \left(\frac{q V}{k T}\right)-1\right] .
$$

Here, $\mu$ is the carrier mobility, $N_{C}$ is the effective density of states in the conduction band and $\mathcal{E}_{\max }$ is the electric field at the Schottky interface. Although there is no change in $\Phi_{B}$ for different thicknesses of IGZO, as shown in Fig. 2b, there is an increase of the gradient of the conduction band minimum $\left(E_{C}\right)$ with reducing thickness. As a result, reducing the thickness leads to an increase in $\mathcal{E}_{\max }$ and a subsequent increase in reverse current, but
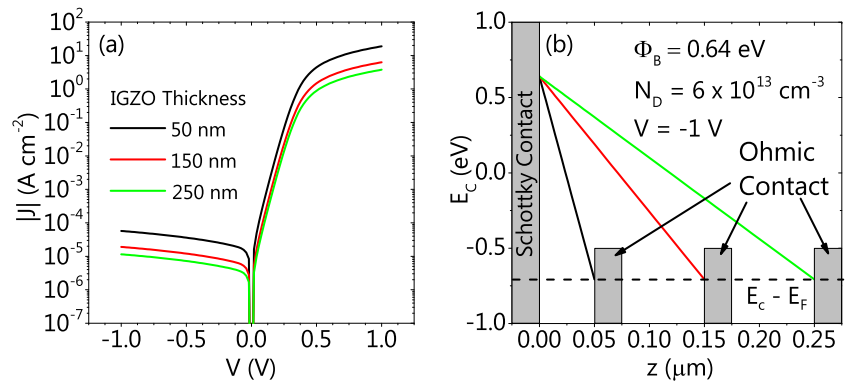

FIG. 2. (a) $|J|-V$ curves for simulated diodes with IGZO thicknesses of 50, 150 and $250 \mathrm{~nm}$. (b) Profiles of the conduction band minimum for simulated diodes with IGZO thicknesses of 50, 150 and $250 \mathrm{~nm}$. 

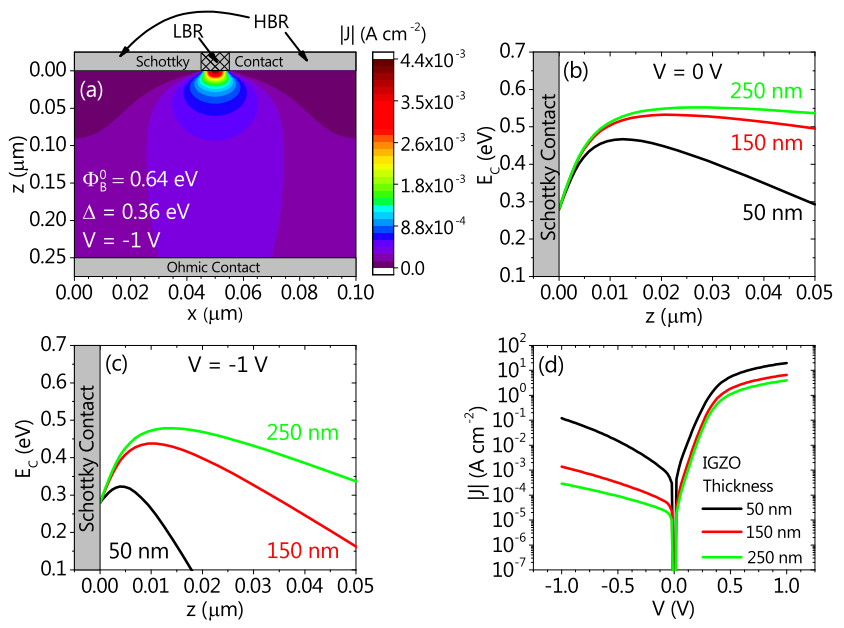

FIG. 3. (a) Contour plot of $|J|$ in a Schottky diode with a LBR in the Schottky contact (cross hatched region) and an IGZO thickness of $250 \mathrm{~nm}$. The diode is under reverse bias $(V=-1 \mathrm{~V})$ and the LBR has a barrier height of $\Phi_{B}=\Phi_{B}^{0}-\Delta=0.28 \mathrm{eV}$. (b) Profiles of $E_{C}$ beneath the LBR at $V=0 \mathrm{~V}$ for Schottky diodes with 50, 150 and $250 \mathrm{~nm}$ thick IGZO layers. (c) The same profiles as in (b) but for $V=-1 \mathrm{~V}$. (d) Corresponding $|J|-V$ curves for the Schottky diodes in (b) and (c).

the magnitude of this increase is much smaller than that observed in Fig. 1a.

Further simulations (not shown here) were carried out to understand the effects of image force lowering, PtIGZO interface roughness and tunnelling. Just as in Fig. 2, the simulated results displayed trends similar to the experimental results, but, importantly, the dependencies lacked the strength of those seen in the experimental results. Thus, we conclude that these phenomena are not the dominant cause of the thickness variation seen in Fig. 1a.

It has been recognised that inhomogeneities in Schottky barrier height can lead to non-ideal $J-V$ characteristics $[26,27]$. A multitude of experimental methods have been used to show that the work function of different crystal faces of the same metal can vary over hundreds of $\mathrm{meV}$ [28-30]. These experimentally observed variations have been corroborated by theoretical studies and ab initio calculations [31, 32]. As the Pt Schottky contact deposited in our experiment is likely to be polycrystalline, different crystal faces will be exposed, leading to variations in the Schottky barrier height. Moreover, in the case of thin-film oxide semiconductors deposited by sputtering there may be local variations in the electron affinity and the density of interface states, further increasing the disorder at the interface. In order to account (at least qualitatively) for such variations in Schottky barrier height, we have included a lower barrier region (LBR) in the centre of the simulated Schottky contact. The LBR is illustrated by the cross hatched region of Schottky contact in Fig. 3a. In Fig. 3 the LBR has a width of $L_{0}=10 \mathrm{~nm}$ and in this region the mean barrier height $\left(\Phi_{B}^{0}\right)$ is reduced by a value of $\Delta=0.36 \mathrm{eV}$. These values for $L_{0}$ and $\Delta$ represent one of a few different circumstances that reflect the large variations in current seen in the experiment while remaining within realistic variations of metal work function as outlined in the literature $[28,29]$. The effects of varying these parameters will be discussed later.

Figure 3a shows a contour plot of $|J|$ for a simulated $250 \mathrm{~nm}$ thick IGZO device for $V=-1 \mathrm{~V}$. It is clear from this image that the reverse current is dominated by the contribution from the LBR. Vertical cutlines of the simulated structures were taken at $x=0.05 \mu \mathrm{m}$ for IGZO thicknesses of 50, 150 and $250 \mathrm{~nm}$ to elucidate the behaviour of $E_{C}$ beneath the LBR. While the $E_{C}$ profiles in the higher barrier regions (HBRs), where $\Phi_{B}=\Phi_{B}^{0}$, display the same behaviour as seen in the homogeneous diode in Fig. 2b, Fig. 3b shows that when $V=0 \mathrm{~V}$, saddle points are formed in the $E_{C}$ profiles below the LBR. These saddle points in $E_{C}$ are formed due to the potential below the HBRs influencing the potential below the LBR $[26,33]$. The maximum value of $E_{C}\left(E_{C M}\right)$ will act as an effective barrier height for the LBR, making it the main determinant of the current. When $V=0 \mathrm{~V}, E_{C M}$ is 0.47 , 0.53 and $0.55 \mathrm{eV}$ for the 50,150 and $250 \mathrm{~nm}$ diodes, respectively. Even at zero bias, the built-in electric field in the $50 \mathrm{~nm}$ diode is much higher than those in the thicker diodes. As such, the thinner diodes will have lower saddle points. A similar trend is displayed in Fig. 3c, where $V=-1 \mathrm{~V}$. In this case, $E_{C M}$ is $0.32,0.44$ and $0.48 \mathrm{eV}$ for the 50, 150 and $250 \mathrm{~nm}$ diodes, respectively. Hence, in the $250 \mathrm{~nm}$ diode, $E_{C M}$ is three-quarters of $\Phi_{B}^{0}$ (the barrier height in the HBR), but in the $50 \mathrm{~nm}$ diode $E_{C M}$ is only half of $\Phi_{B}^{0}$. As the reverse current is proportional to $\exp \left(-\frac{E_{C M}}{k T}\right)$, the strong thickness dependence of $E_{C M}$ explains the orders of magnitude differences in reverse current seen in the corresponding $|J|-V$ curves in Fig. 3d. Furthermore, changing the bias from $V=0 \mathrm{~V}$ to $V=-1 \mathrm{~V}$ leads to changes in $E_{C M}$ of $0.14,0.09$ and $0.07 \mathrm{eV}$ for the 50, 150 and $250 \mathrm{~nm}$ cases, respectively. Thus, $E_{C M}$ in thinner diodes is more easily reduced by increasing reverse bias, which explains the steeper gradients of the reverse current in thinner diodes seen in Fig. 3d. Similar changes in $E_{C M}$ in forward bias can be used to explain the thickness dependencies of extracted values of $\Phi_{B}$ and $n$, but they are far less dramatic.

The simulation results in Fig. 3 qualitatively reproduce the trends seen in the experiments in Fig. 1a and in $[14,21]$, particularly the orders of magnitude differences in reverse current. Exact fitting of the experimental results is hampered by the simplicity of the model. Firstly, the use of two-dimensional simulations constrains the accuracy of results as the LBR is only pinched off in one dimension. Furthermore, real diodes are likely to contain many inhomogeneities with a broad distribution of areas and barrier heights dependent on various factors including which Pt crystal faces are exposed and the amorphous nature of IGZO. Our simulations (not shown here) 

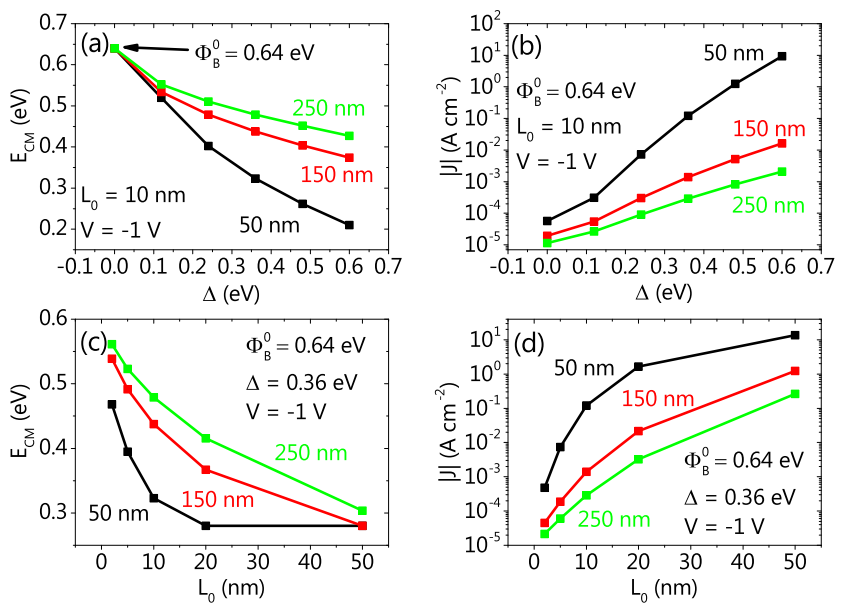

FIG. 4. Effects of variations in inhomogeneity magnitude and size upon the reverse biased $E_{C}$ profiles and current densities of Schottky diodes with IGZO thicknesses of 50, 150 and $250 \mathrm{~nm}$. (a) $E_{C M}$ against $\Delta$ for $L_{0}=10 \mathrm{~nm}$ and $V=-1 \mathrm{~V}$. (b) $|J|$ against $\Delta$ for $L_{0}=10 \mathrm{~nm}$ and $V=-1 \mathrm{~V}$. (c) $E_{C M}$ against $L_{0}$ for $\Delta=0.36 \mathrm{eV}$ and $V=-1 \mathrm{~V}$. (d) $|J|$ against $L_{0}$ for $\Delta=0.36 \mathrm{eV}$ and $V=-1 \mathrm{~V}$.

indicate that when multiple inhomogeneities are present the behaviours seen in Fig. 3 are preserved. In fact, if the LBRs are are close enough together to influence each other the value of $E_{C M}$ below the LBRs is further reduced.

To account for different experimental conditions, we have considered the effects of varying $\Delta$ and $L_{0}$ upon $E_{C M}$ and reverse current. The value of $\Delta$ was varied from 0 to $0.60 \mathrm{eV}$ in $0.12 \mathrm{eV}$ steps, while five different values of $L_{0}$ were used: 2, 5, 10, 20 and $50 \mathrm{~nm}$. Figure 4 demonstrates that increasing the size and reducing the barrier height of the LBR will give rise to lower values of $E_{C M}$ and lead to significantly larger current densities in reverse bias. Such trends have been discussed previously in the case of Schottky diodes with conventional semiconductor layers much thicker than the depletion width $[26,33]$, however, Fig. 4 further demonstrates such influence is far more pronounced in fully depleted thin-film diodes.

Figure 4a shows that for fully depleted thin-film diodes, increasing $\Delta$ has a far more significant effect on the $E_{C M}$ of thinner devices. At low values of $\Delta$, there is a very weak dependence of $E_{C M}$ on IGZO thickness, but as
$\Delta$ increases this thickness dependence grows stronger. Hence, the thickness dependence of the reverse current will be exacerbated at higher values of $\Delta$, as shown in Fig. 4b. Given the orders of magnitude differences in reverse current seen in Fig. 1a, it is clear that our results are more closely described by large values of $\Delta$, i.e., large variations in barrier height.

Figures $4 \mathrm{c}$ and $\mathrm{d}$ show that in the limit where $L_{0}$ is very small, the thickness dependence of $E_{C M}$ also begins to become small, hence the thickness dependence of the reverse current is limited. As $L_{0}$ gets larger the thickness dependence strengthens, but at very large values of $L_{0}$ the transport will tend to a limit where the HBR and LBR effectively conduct in parallel. This parallel conduction will lead the reverse current to behave as if it were a low barrier diode with barrier height $\Phi_{B}=\Phi_{B}^{0}-\Delta$ and an area equal to that of the LBR. In this limit there will be a weak thickness dependence akin to the one shown in Fig. 2. Thus, as our results display a very strong thickness dependence of the reverse current, they are unlikely to be in either of these limiting cases.

In summary, we have identified and explained a strong thickness dependence of the current in thin-film Schottky diodes for semiconductor thicknesses less than the depletion width. Most notable is the dramatic increase in reverse current for thinner semiconductor layers. Simulation results indicate that the main determinants of the current are saddle points in $E_{C}$ formed due to inhomogeneities in Schottky barrier height. These saddle points are strongly dependent on semiconductor thickness and bias. Simulation results also demonstrated that thinner diodes are more sensitive to variations in the magnitude and size of inhomogeneities, leading to greater reverse currents. These findings may have implications not only for diode design and optimisation, but also for other device structures, such as Schottky barrier transistors and MESFETs based on IGZO and other thin-film semiconductors.

We are grateful for the technical support from M. McGowen, I. Hawkins and L. Zhang. This work was supported by North-West Nanoscience Doctoral Training Centre, EPSRC grants (EP/N021258/1 and EP/G03737X/1), National Key Research and Development Program of China (2016YFA0301200 and 2016YFA0201800), National Natural Science Foundation of China (11374185 and 11304180).
[1] T. Georgiou, R. Jalil, B. D. Belle, L. Britnell, R. V. Gorbachev, S. V. Morozov, Y.-J. Kim, A. Gholinia, S. J. Haigh, O. Makarovsky, et al., Nat. Nanotechnol. 8, 100 (2013).

[2] S. Ju, A. Facchetti, Y. Xuan, J. Liu, F. Ishikawa, P. Ye, C. Zhou, T. J. Marks, and D. B. Janes, Nat. Nanotechnol. 2, 378 (2007).

[3] C. D. Dimitrakopoulos and D. J. Mascaro, IBM J. Res.
Dev. 45, 11 (2001).

[4] J.-H. Lee, D.-H. Kim, D.-J. Yang, S.-Y. Hong, K.-S. Yoon, P.-S. Hong, C.-O. Jeong, H.-S. Park, S. Y. Kim, S. K. Lim, et al., in SID Symposium Digest of Technical Papers, Vol. 39 (Wiley Online Library, 2008) pp. 625-628.

[5] K. Nomura, H. Ohta, A. Takagi, T. Kamiya, M. Hirano, and H. Hosono, Nature 432, 488 (2004).

[6] H. Yabuta, M. Sano, K. Abe, T. Aiba, T. Den, H. Ku- 
momi, K. Nomura, T. Kamiya, and H. Hosono, Appl. Phys. Lett. 89, 112123 (2006).

[7] E. Fortunato, P. Barquinha, and R. Martins, Adv. Mater. 24, 2945 (2012).

[8] T. Kamiya, K. Nomura, and H. Hosono, Sci. Tech. Adv. Mater. 11, 044305 (2010).

[9] D. H. Lee, K. Nomura, T. Kamiya, and H. Hosono, IEEE Electron Device Lett. 32, 1695 (2011).

[10] H. Kim, S. Kim, K.-K. Kim, S.-N. Lee, and K.-S. Ahn, Jpn. J. Appl. Phys 50, 105702 (2011).

[11] A. Chasin, S. Steudel, K. Myny, M. Nag, T.-H. Ke, S. Schols, J. Genoe, G. Gielen, and P. Heremans, Appl. Phys. Lett. 101, 113505 (2012).

[12] A. Chasin, M. Nag, A. Bhoolokam, K. Myny, S. Steudel, S. Schols, J. Genoe, G. Gielen, and P. Heremans, IEEE Trans. Electron Dev. 60, 3407 (2013).

[13] J. Zhang, H. Wang, J. Wilson, X. Ma, J. Jin, and A. Song, IEEE Electron Device Lett. 37, 389 (2016).

[14] J. Zhang, Y. Li, B. Zhang, H. Wang, Q. Xin, and A. Song, Nat. Commun. 6 (2015).

[15] M. Lorenz, A. Lajn, H. Frenzel, H. v. Wenckstern, M. Grundmann, P. Barquinha, R. Martins, and E. Fortunato, Appl. Phys. Lett. 97, 243506 (2010).

[16] T. T. Trinh, V. D. Nguyen, H. H. Nguyen, J. Raja, J. Jang, K. Jang, K. Baek, V. A. Dao, and J. Yi, Appl. Phys. Lett. 100, 143502 (2012).

[17] A. Chasin, L. Zhang, A. Bhoolokam, M. Nag, S. Steudel, B. Govoreanu, G. Gielen, and P. Heremans, IEEE Electron Device Lett. 35, 642 (2014).

[18] A. Chasin, V. Volskiy, M. Libois, K. Myny, M. Nag, M. Rockelé, G. A. Vandenbosch, J. Genoe, G. Gielen, and P. Heremans, IEEE Trans. Electron Dev. 61, 3289
(2014).

[19] M. D. Jacunski, M. S. Shur, A. A. Owusu, T. Ytterdal, M. Hack, and B. Iniguez, IEEE Trans. Electron Dev. 46, 1146 (1999).

[20] A. Valletta, P. Gaucci, L. Mariucci, G. Fortunato, and S. Brotherton, Appl. Phys. Lett. 85, 3113 (2004).

[21] Q. Xin, L. Yan, Y. Luo, and A. Song, Appl. Phys. Lett. 106, 113506 (2015).

[22] H. Mosbacker, Y. Strzhemechny, B. White, P. Smith, D. C. Look, D. Reynolds, C. Litton, and L. Brillson, Appl. Phys. Lett. 87, 012102 (2005).

[23] J. Zhang, Q. Xin, and A. Song, J. Vac. Sci. Technol. A 34, $04 \mathrm{C} 101$ (2016).

[24] S. M. Sze and K. K. Ng, Physics of Semiconductor Devices, 3rd ed. (John Wiley \& Sons Inc., New York, U.S.A., 2006).

[25] T.-C. Fung, C.-S. Chuang, C. Chen, K. Abe, R. Cottle, M. Townsend, H. Kumomi, and J. Kanicki, J. Appl. Phys. 106, 084511 (2009).

[26] R. Tung, Phys. Rev. B 45, 13509 (1992).

[27] J. H. Werner and H. H. Güttler, J. Appl. Phys. 69, 1522 (1991).

[28] R. Strayer, W. Mackie, and L. Swanson, Surf. Sci. 34, 225 (1973).

[29] M. Chelvayohan and C. Mee, J. Phys. C 15, 2305 (1982).

[30] N. Gaillard, D. Mariolle, F. Bertin, M. Gros-Jean, M. Proust, A. Bsiesy, A. Bajolet, S. Chhun, and M. Djebbouri, Microelectron. Eng. 83, 2169 (2006).

[31] R. Smoluchowski, Phys. Rev. 60, 661 (1941).

[32] H. L. Skriver and N. Rosengaard, Phys. Rev. B 46, 7157 (1992).

[33] J. Sullivan, R. Tung, M. Pinto, and W. Graham, J. Appl. Phys. 70, 7403 (1991). 